\title{
Canon y corpus literario latinoamericano y caribeño. Una metodología de construcción del corpus
}

Adrián R. Vila

Universidad de Buenos Aires, Argentina

Universidad de Salamanca, España

editorvila@gmail.com

Cita sugerida: Vila, A. R. (2018). Canon y corpus literario latinoamericano y caribeño. Una metodología de construcción del corpus. Palabra Clave (La Plata), 7(2), e050. https://doi.org/10.24215/18539912e050 


\section{Canon y corpus literario latinoamericano y caribeño. Una metodología de construcción del corpus}

Canon and Latin American and Caribbean literary corpus. A corpus building methodology

Adrián R. Vila

Universidad de Buenos Aires, Argentina

Universidad de Salamanca, España

editorvila@gmail.com

\section{Resumen:}

El presente trabajo tiene como objeto la presentación de un modelo metodológico para la construcción de un corpus literario latinoamericano y caribeño. Esta metodología se plantea, asimismo, como un modelo para ser replicado para otros objetos de investigación dado su diseño basado en formas textuales sistémicas y extrasistémicas, canónicas y no canónicas. Se presentan algunos resultados obtenidos en búsquedas en plataformas y bibliotecas digitales de los títulos del corpus diseñado. En este punto destacamos que la eficacia del corpus a diseñar se mensura en la posibilidad de dar respuestas a algunos de los interrogantes planteados como estrategia de construcción: “¿Corpus de qué?", “¿Corpus para qué?", "¿Qué resultados nos permite obtener su diseño?".

Palabras Clave: Metodología, Corpus literario, Libros electrónicos, Soporte impreso.

\section{Abstract:}

This paper aims at presenting a methodological model for the building of a Latin American and Caribbean literary corpus. This methodology is also presented as a model to be replicated to other objects of investigation given its systemic and based on nonsystemic, canonical and non-canonical textual forms design. Some results of search platforms and digital libraries of titles designed corpus presented. At this point we emphasize that the effectiveness of design corpus is surveying the possibility of providing answers to some of the questions raised as construction strategy is: "Corpus what else?", "Corpus why?", "What results allow us to design?".

KEYWORDS: Methodology, Literary corpus, E-books, Paper work.

\section{A) INTRoDUCción}

La categoría América Latina designa, desde una perspectiva geográfica, las partes del nuevo mundo que fueron colonizadas por naciones de la Europa latina como España, Francia y Portugal. Pero también existen zonas del continente americano que fueron dominadas por Inglaterra u Holanda. Asimismo, hay zonas de Canadá y EE. UU. que fueron colonizadas por Francia y España, pero no se consideran latinoamericanas. Para analistas como Rouquié (1989, p. 17), América Latina sería un fallido "concepto cultural”, que contendría a las naciones de cultura latina de América. Este tipo de encuadres traerían aparejadas otras inconsistencias como la inclusión de Canadá y su provincia francohablante de Quebec, "infinitamente más latina que Belice y tanto como Puerto Rico" estado libre asociado de EE. UU., pero a los que nunca nadie ha pensado incluirlos, "ni siquiera al nivel de su provincia francohablante, en su subconjunto latinoamericano" (Rouquié, 1989, p. 17). A su vez, destacamos las imprecisiones y la ambigüedad de la etiqueta América Latina al dilucidar que este concepto no es "ni plenamente cultural ni solamente geográfico" (Rouquié, 1989, p. 17). Entonces, utilizaremos la categoría América Latina porque se ofrece como término "cómodo", sin ignorar "sus límites y sus ambigüedades" (Rouquié, 1989, p. 20). Si las organizaciones globales como ONU, UNESCO y Banco Mundial imponen la categoría América Latina y el Caribe con fines de simplificación 
estadística, la composición de la Comunidad de Estados Latinoamericanos y Caribeños (CELAC) demuestra que estos países la aceptan. Es decir que el "autorreconocimiento de la etiqueta por los países implicados vía participación en la CELAC” (Malamud, 2016) funciona como uno de los criterios de demarcación. A esta vinculación entre América Latina y el Caribe en términos de políticas gubernamentales le es subsidiaria la vinculación de las literaturas de todos los sistemas literarios que componen la región caribeña con los sistemas que componen la literatura latinoamericana. La vinculación teórica entre esos dos bloques fue articulada, sobre todo, por dos tipos de dispositivos teóricos: 1) por un lado, la historia común del Caribe insular anclada en rasgos económico-sociales similares y, por otro lado, 2) las relaciones históricas comunes del Caribe con zonas de la región latinoamericana, las formas de desarrollo cultural similares y la literatura, por la evidencia de temas y problemas. A su vez, estos rasgos de similitud en la organización de los sistemas literarios de América Latina y Caribe son expuestos por aquellos trabajos de este tipo de dispositivos teóricos del tipo 2) que marcábamos previamente: a) sistemas literarios ligados a la metrópoli colonial (Gran Bretaña, Holanda, Francia); b) sistemas literarios en créole, papiamento, papiamen, etc., y c) sistemas literarios multilingües. En este punto, la organización de los sistemas literarios del Caribe, Antillas y Guayanas, se comporta en relación con las estructuras lingüísticas que dejó la metrópolis de la misma manera que en América Latina.

Las operaciones de integración literaria (y cultural) latinoamericana (y luego latinoamericana y caribeña) se iniciaron con un nivel inicial de integración de las letras en castellano, luego pasaron a un segundo nivel de integración de las letras americanas en castellano y portugués (sumando a la literatura brasileña), para derivar en un tercer nivel de integración de las literaturas de todas las lenguas latinas con la inclusión de las literaturas en francés (especialmente, de Haití). Ardao (1996, pp. 33-34) propone un nivel de integración "supernumerario" con la inclusión de "las letras americanas de otras lenguas correspondientes a su área: autóctonas hacia un extremo, de procedencia europea no latina hacia otro". En este cuarto nivel de integración, tendríamos un sistema literario compuesto por todas las literaturas en todas las lenguas de todas las regiones de América Latina, Caribe, Antillas y Guayanas.

Efectuada esta delimitación terminológica, se procedió a la realización de un corpus literario latinoamericano y caribeño (CLLyC) que representara a los sistemas literarios mencionados. El CLLyC se desarrolla en función de dilucidar la relación entre literatura latinoamericana y caribeña e industria editorial en digital. Es decir, una zona de investigación a partir del análisis de la transposición a los distintos formatos en digital de estos títulos "no nativos digitales" (no producidos especialmente por medios digitales) (Strickland, 2009), tomando como muestra el segmento latinoamericano del canon realizado por Harold Bloom (1995) y ampliado en un corpus de segmentos de autores y títulos provistos por aquellas corrientes teóricas distantes y en tensión con el mainstream literario.

\section{B) Metodología del corpus}

El Corpus literario latinoamericano y caribeño (CLLyC) desarrollado se asienta en fuentes documentales literarias latinoamericanas múltiples, opuestas, complementarias, tanto en el tiempo en el que se escribieron las obras o en que vivieron sus autores o por la edad de ellos, como en los criterios de elección de autores y títulos como por los repertorios adoptados. Así, fueron empleados repertorios, ensayos, antologías, arti\#culos académicos, diccionarios, y se desarrollaron segmentos enteros a partir de la combinación de varios de ellos. Los listados de obras resultantes fueron desarrollados a partir de fuentes que las aportan desde enfoques diversos y se desempeñan metodológicamente de manera complementaria para las búsquedas realizadas, ocupan las áreas de vacancia que dejan las demás fuentes o, en algunos casos, se yuxtaponen en parte. Y, también, funcionan metodológicamente como opuestos entre sí, lo que permite expresar al interior del CLLyC la complejidad de las formas literarias de la región y las dispositividades materiales (Chartier, 2006) mediante las cuales circulan esas literaturas. Así, el corpus propuesto comprende tanto al segmento latinoamericano y caribeño El canon occidental de Bloom (1995), como a prácticas discursivas y 
culturales que incorporen la hibridez de la producción latinoamericana, las voces femeninas de su literatura, las voces de la producción precolombina, las literaturas periféricas, las heteroglosias que componen su campo cultural y literario, las distintas maneras que tienen las voces subalternas de su literatura, las literaturas indígenas, las voces queer y feministas, las literaturas que se expresan en otras lenguas que no son el español y el portugués (ni el francés). Con una estrategia de diseño general que exhiba la mayor cantidad posible de perspectivas sobre/desde la literatura latinoamericana y caribeña en el marco de procesos que reconocemos como ilimitados (las incorporaciones desde distintas perspectivas sobre la literatura latinoamericana y caribeña pueden tornarse infinitas). Entonces se procedió al diseño del CLLyC (Figura 1) de acuerdo con los siguientes pasos y criterios: se construyó la lista de 18 autores y 36 obras que Harold Bloom introduce en el segmento latinoamericano de su canon, y los cinco autores y siete obras que introduce en el segmento caribeño (West Indian). Es decir: 43 títulos de 23 autores de El canon occidental de Harold Bloom (1995).

El Diccionario de autores latinoamericanos de Aira (2001) aporta más de 1.300 autores y 11.102 obras con un criterio de amplitud en la incorporación de dramaturgos, poetas, novelistas, cuentistas, científicos, historiadores, cronistas y ensayistas. Asimismo, nos provee de un segmento de literatura brasileña. No incluye autores y obras precolombinos ni en otras lenguas que no sean castellano y portugués, ni autores surgidos a partir de 1985. A su vez, el Diccionario de la literatura latinoamericana de Cella (1998) contribuye con un listado de autores y obras que funciona de manera complementaria: incorpora textos precolombinos y en lenguas no hispánicas y a los escritores nacidos hasta 1940 o, en caso de que hayan nacido después del 40, que ya estuviesen fallecidos en 1998. Aporta 4.737 títulos de 595 autores. La idea de descolonización de la literatura latinoamericana propone la recuperación de los cánones literarios propios de las comunidades amerindias, que la acción de canonización basada en "la lengua y en los valores de las culturas colonizadoras más importantes" logró silenciar, pero no suprimir (Mignolo, 1998, p. 241). Así, se elaboró un segmento específico para que la literatura precolombina esté representada en el CLLyC. Este segmento elaborado aporta 50 títulos de literaturas precolombinas y 8 autores (muchas de las obras son anónimas).

Saraus. Movimiento - Literatura - Periferia - São Paulo. Antología, de Lucía Tennina (2014) aporta autores y obras de poetas paulistas de la llamada "marginalia paulista". Aporta 58 títulos de 38 autores. De la Tricolor a la Wiphala. Narrativa contemporánea de Bolivia, de Di Nucci, Recoaro y Grieco y Bavio (2014a) contribuye con cuentos de autores nacidos entre 1956 y mediados de los 80, con la que articula una selección que expresa la emergencia de una nueva narrativa boliviana independiente. Aporta 67 títulos de 14 autores.

McOndo (una antología de nueva literatura hispanoamericana), de Fuguet y Gómez (1996) aporta a este CLLyC cuentos de aquellos autores incorporados a la antología nacidos desde 1959 "(que coincide con la siempre recurrida revolución cubana) a 1962 (que en Chile y en otros países, es el año en que llega la televisión)" (Fuguet y Gómez, 1996, p. 9) y cuya selección descarta todo rastro de realismo mágico como poética. Ese listado de autores y de obras resultante de la antología McOndo es el aporte a este CLLyC: 17 títulos de 15 autores. El Manifiesto del Crack (Palou, Urroz, Padilla, Chávez-Castañeda, y Volpi, 2000) aporta una lista de seis autores mexicanos (Jorge Volpi, Pedro Ángel Palou, Eloy Urroz, Ignacio Padilla, Ricardo Chávez Castañeda y Vicente Herrasti), con un programa poético de una narrativa dislocada del espacio y del tiempo de México, cuyas obras, posteriores a 1996, se caracterizan por una postura narrativa rupturista tanto del realismo mágico latinoamericano como de una cierta poética light. Aporta 22 títulos de seis autores. Si los cuentos elegidos para $\mathrm{McOndo} \mathrm{también} \mathrm{promulgan} \mathrm{una} \mathrm{postura} \mathrm{narrativa} \mathrm{rupturista} \mathrm{con} \mathrm{el} \mathrm{realismo}$ mágico latinoamericano, Carlos Fuentes (2011) en La gran novela latinoamericana nos brinda 124 títulos de 65 autores desde la perspectiva de abordaje de un escritor del boom.

Si el Diccionario de Aira (2001) propone autores y obras en español y portugués, Los chongos de Roa Bastos. Narrativa contemporánea de Paraguay de Di Nucci, Recoaro y Grieco y Bavio (2014b) y Saraus aportan en un caso obras en jorapa, guaraní y distintas formas de heteroglosia e hibridaciones entre guaraní, castellano y otras lenguas y hablas indígenas y, en otro caso, textos en el portugués de los jóvenes de la perifatividade paulista. Aporta 33 títulos de nueve autores. 
Latin American Women Writers aporta 2.951 obras de 303 escritoras nacidas entre los siglos XVI y fines del siglo XX. Los chongos de Roa Bastos y Saraus contribuyen con obras de autores nacidos en el periodo de 1965 y 1984 en el primer caso, De la Tricolor a la Wiphala propone autores nacidos entre 1956 y mediados de los 80; en el caso de Saraus, aporta autores nacidos entre 1957 y mediados de los 80, que en su mayoría inician sus trabajos literarios en las décadas del 90 y principios de los 2000; así como McOndo (una antología de nueva literatura hispanoamericana) aporta escritores nacidos entre 1959 y que se destacan en su trabajo literarios a mediados de los 90. La antología 1.000 millones: poesía en lengua española del siglo XXI (Helder, Henderson, y Orge, 2014) incluye 33 autores hispanoamericanos nacidos entre 1980 y 1995, y el Manifiesto del Crack (Palou, Urroz, Padilla, Chávez-Castañeda, y Volpi, 2000) incorpora seis autores mexicanos nacidos entre 1961 y 1968 y cuyas obras son todas posteriores a 1996. Asimismo, la selección de Bogotá39 (s.f.) selección realizada por los escritores colombianos Piedad Bonnet, Héctor Abad Faciolince y Óscar Collazosaporta 133 obras de 39 autores menores de 39 años en el año 2007.

Si el Manifiesto del Crack propone novelas que faciliten su traducción, Los chongos de Roa Bastos y Saraus aportan cuentos con complejidades textuales de difícil traducción, dada la utilización poética de heteroglosia y variedades dialectales del castellano, del guaraní, del portugués y del jorapa, entre otras lenguas indígenas y, por otro lado, las formas del habla del portugués de los jóvenes y sectores subalternos de los barrios periféricos de San Pablo. De la Tricolor a la Wiphala contribuye con cuentos escritos en las distintas variedades del castellano de Bolivia (excepto en el caso de Alison Spedding, que escribe en aymará). El ensayo Latin American Writers on Gay and Lesbian Themes: A Bio-Critical Sourcebook, de David W Foster (1991), colabora con textos escritos en español y en portugués, aunque el autor trabaja con obras en español y portugués y obras traducidas al inglés. La selección Bogotá39 (s.f.) incluye autores que escriben en español, portugués e inglés.

Mientras la mayoría de las fuentes utilizadas no destaca una perspectiva de género, los repertorios Latin American Women Writers (André y Bueno, 2008) y Latin American Writers on Gay and Lesbian Themes: A Bio-Critical Sourcebook lo hacen desde las diversas narrativas propuestas por escritoras latinoamericanas en el primer caso y, en el segundo caso, con la propuesta de 27 títulos de 25 autores que nos permiten incluir en el CLLyC las perspectivas que articulan la relación entre homosexualidad y sociedad en la literatura latinoamericana.

Si McOndo y De la Tricolor a la Wiphala proponen cuentos, el Manifiesto del Crack propone novelas, al igual que la propuesta de Carlos Fuentes (2011) en la Gran novela latinoamericana; Saraus propone poesías, al igual que la antología 1.000 millones.

Una sección de títulos de escritores caribeños, antillanos y de las Guayanas diseñado sobre la base de la combinación de los títulos y escritores que componen las siguientes fuentes: West Indian Narrative: An Introductory Anthology (Ramchand, 1996); Jahaji Bhai: An Anthology of Indo-Caribbean Literature (Birbalsingh, 1988); They Came in Ships: An Anthology of Indo-Guyanese Writing (Benjamin, Kallicharan, McDonald y Searwar, 1998); The Routledge Reader in Caribbean Literature (Donnell y Lawson Welsh, 1996); Sugar Is All: Caribbean Short Stories and Poems from St. Kitts and Nevis (Walwyn, 2001); Caribbeana: An Anthology of English Literature of the West Indies, 1657-1777 (Krise, 1999); The Companion to Anglophone Caribbean Literature (Donnell y Bucknor, 2011); The Oxford Book of Caribbean Verse (Brown y McWatt, 2004) y los escritores caribeños, antillanos y de Guayanas de la Encyclopedia of Latin American and Caribbean Literature 1900-2003 (Balderston y Gonzalez, 2004). Esta sección aporta 2.597 títulos de 559 autores. La radicalidad de la incorporación de este segmento se enmarca en la idea de que la articulación de "lo caribeño" es una unidad "inseparable de América Latina" (Pizarro, 1987, p. 146). La inclusión de estos títulos habilita la descripción de sistemas literarios caribeños ligados a las distintas metrópolis (en castellano, portugués, francés, holandés); la descripción de sistemas literarios en créole, patois, papiamento, neerlandes y a los sistemas literarios multilingües en algunas de las expresiones literarias incorporadas, tal cual se plantea en la "Introducción". 
La incorporación de los títulos de autores latinoamericanos y caribeños del Catálogo de Editoriales Cartoneras latinoamericanas de la Universidad de Wisconsin (University of Wisconsin, s.f.) nos permite cruzar lo que Chartier (2006, p. 10) proponía para la sociología de los textos: no disociar "el análisis de las significaciones simbólicas del de las formas materiales que las transmiten”. Es decir que la dispositividad material, mediante la cual circulan los textos de las literaturas latinoamericanas que editan, se organiza como especificidad editorial a través de la cual circulan formas literarias propias de Latinoamérica y el Caribe, lo que Hall (1991, p. 37) sintetizaba como la revolución cultural más profunda, resultado del ingreso de los márgenes a la representación "en la pintura, en el cine, en la música, en la literatura, en las artes modernas de todos lados, en política y, en términos generales, en la totalidad de la vida social". Este segmento de editoriales cartoneras aporta 469 títulos de 358 autores.

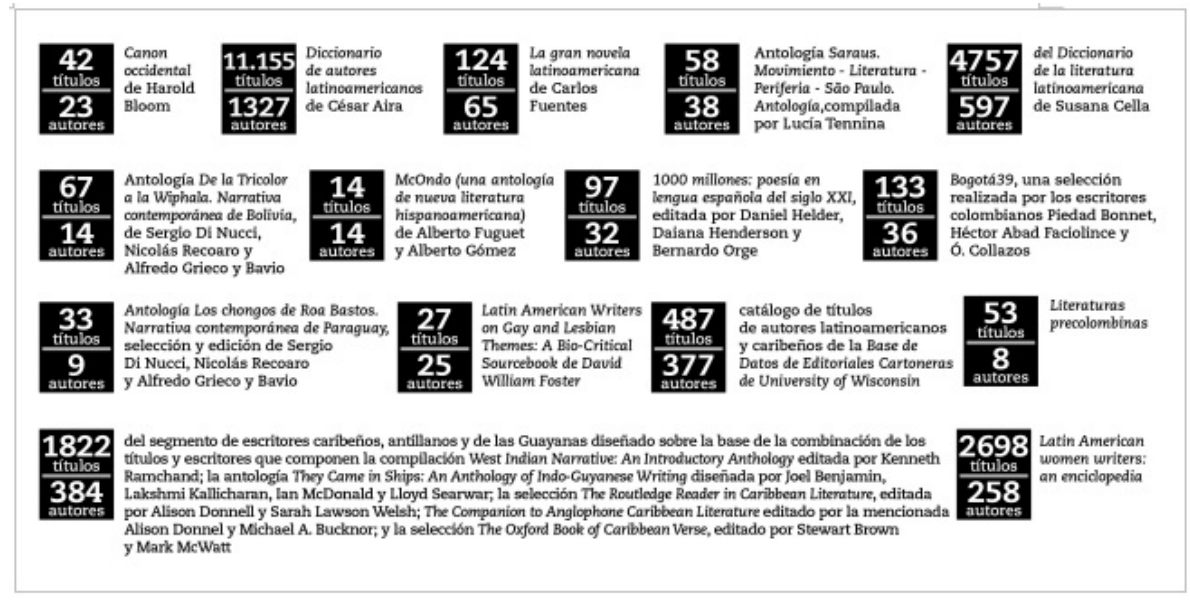

FIGURA 1

Composición del CLLyC: títulos y autores aportados por cada segmento elaboración propia

Con el objetivo de relevar la transposición de las obras del CLLyC a formato digital, se realizó la búsqueda de los mencionados títulos en dicho formato en 14 de las plataformas comerciales y en una serie de ocho bibliotecas destacadas desde el punto de vista lingüístico y que sobresalen por prestaciones, arquitectura y disponibilidad digital (Chowdhury y Foo, 2012; Klibanski, 2006; Tramullas Saz, 2002; Turiel, 2010). Amazon es, de hecho, la empresa número uno del mercado de libros electrónicos con más de 500.000.000 unidades vendidas (el 82\% tomando en cuenta las ventas en EE. UU., Reino Unido, Canadá y Australia) (Author Earnings, 2017). Kindle Unlimited posee un catálogo de 4.600 .000 de títulos (Ramírez, 2016). Su competidora Barnes \& Noble tiene casi 3.000.000 de títulos de libros digitales. iBookstore y iTunes poseen una media de ventas mensuales de 17.000.000 de unidades (Dedieu, 2013). Google Books ofrece sus servicios de casi 350.000 títulos y su servicio Play Books ofrece más de 4.000.000 de títulos. Kobo es una plataforma canadiense de libros electrónicos de estándares abiertos, que alcanza a 10.000.000 de usuarios y posee un catálogo de más de 5.000.000 de libros (Ramírez, 2016). La selección de Free-eBooks se debe a la necesidad de obtener el estado de la oferta en digital de plataformas de descarga gratuitas. Scribd se presenta como un sistema de intercambio de archivos y acceso a libros electrónicos, con un catálogo de 700.000 libros. Nubico cuenta con 100.000 clientes y dispone de más de 20.000 títulos. Livraria Cultura, Gandhi y Librería Santa Fe son plataformas destacadas en el comercio editorial latinoamericano. En el nivel de las bibliotecas, Public Library New York y Columbia University Libraries son bibliotecas prestigiosas a nivel internacional por la disposición de múltiples recursos en digital. La Biblioteca Nacional de España es la biblioteca más reconocida en el campo idiomático del castellano, así como Gallica - Biblioteca Nacional de Francia es la más reconocida en lengua francesa. Europeana es una biblioteca digital que posee casi 4.000.000 de objetos digitales de la Unión Europea. La Biblioteca del 
Instituto Latinoamericano de Berlín se seleccionó como biblioteca de un organismo especializado en temas latinoamericanos. Las bibliotecas Nacional de Perú y de San Pablo forman parte del segmento de bibliotecas latinoamericanas con mayor disposición de colecciones en digital del área geográfica.

Para la realización de las búsquedas, se diseñó un bot de recolección de resultados de estas. Luego, se cargaron los campos correspondientes a autor, título, fuente del CLLyC y se procedió a la búsqueda en cada plataforma y biblioteca, verificando los resultados por criterios de coincidencia. Finalmente, se crearon tablas con los datos recolectados y se obtuvo una base de datos con más de dos millones de registros (por tuplas compuestas por sitio Web, título buscado, autor buscado, título encontrado, soporte -impreso y digital-y autor encontrado). Se realizaron, asimismo, verificaciones manuales como método de validación de estas: se hicieron, en total, 61.551 búsquedas de autores y 207.570 búsquedas de autor + libro. Todas las búsquedas se hicieron en el idioma original de las obras.

\section{c) Resultados y discusión}

El presente trabajo propone definir una metodología de construcción de un corpus literario para la realización de búsquedas de títulos y autores, en el marco de una investigación del estado del arte de la transposición de impreso a digital de literatura latinoamericana y caribeña. Ahora bien: ¿cómo construir el corpus literario latinoamericano y caribeño (CLLyC) al que nos referimos? Lotman (2000) propone una descripción del funcionamiento de los sistemas culturales como poseedores de una dualidad que consiste en la articulación de dos tendencias dinámicas inversas: por un lado, una tendencia a la diversidad y, por el otro, una tendencia a la uniformidad. Entonces, tomando como préstamo esta perspectiva para la construcción de nuestro CLLyC, este puede ofrecerse como un sistema compuesto de múltiples subsistemas diversos e intercambiables. En este nivel, el CLLyC diseñado pone de manifiesto lo que Lotman (2000, p. 209) define como "tendencia [de la cultura] a la multiplicación de lenguajes diversos". Al diseñarse un corpus desde una concepción semiótica del campo cultural, es decir, de coexistencia en puja de tendencias enfrentadas, se facilita tanto la inclusión como la supresión de segmentos que lo constituyen. Asimismo, la noción de inestabilidad (Eagleton, 1998, p. 24) de una definición de qué es literatura en cada momento histórico, y los juicios de valor sobre esos "discursos" en cada circunstancia sociohistórica, también sería uno de los principales vectores de apreciación de la configuración del CLLyC diseñado. Pero, al mismo tiempo, el propio sistema genera formas de autodescripción, a las que Lotman llama metadescripciones, que funcionan como modelos en la necesidad de describirse a sí mismo y que produce elecciones de tipos de textos “mejores” para esa descripción necesaria de la mano de "la pluma de los críticos, los teóricos, los legisladores del gusto y, en general, de los legisladores” (Lotman, 1996, p. 75) en ese plano se desarrollaría la función canónica. Si el propio desarrollo de la cultura está asentado sobre la puja entre las formas sistémicas y las extrasistémicas, en el corpus desarrollado como CLLyC estas tensiones se expresan sin exclusiones en el universo resultante obtenido de 20.409 títulos diferentes de 2.932 autores de 43 nacionalidades. De ese universo, fueron hallados en impreso 14.835 (casi el $75 \%$ del corpus buscado). De ese total de títulos hallados en impreso, el 50,62\% ha sido transpuesto a digital, 6.447 títulos (cerca del 30\% del corpus inicial buscado), pero existen 12.071 títulos (casi el 80\% de los hallados en algún soporte en alguna plataforma o biblioteca) que solamente se encuentra en impreso. En digital, la plataforma que más títulos ofrece es Scribd: 1.718 títulos (35,48\% de los títulos existentes en digital). Amazon ofrece 1.101 títulos, es decir, una oferta media (el 22,73\%). Livraria Cultura ofrece 1.035 (21,37\% de los títulos disponibles); Barnes \& Noble, 600 títulos en digital (12,39\%). El resto de la oferta en digital en plataformas es baja. En relación con las bibliotecas, la Biblioteca Nacional de España es la que mayor cantidad de títulos ofrece en digital: 1.749 (el 36,12\% de la oferta disponible en digital). Este dato es muy destacable, dado que la Biblioteca Nacional de España ofrece en digital más títulos que las demás plataformas y bibliotecas en las que se efectuaron las búsquedas (incluidos Scribd y la plataforma Amazon). 
Luego la siguen la Biblioteca Europeana de la Unio\#n Europea, con 582 títulos (12,01\%) y Columbia University Libraries con 572 títulos $(11,81 \%)$. El resto de la oferta en digital en bibliotecas es muy baja (Figura 2).

\begin{tabular}{|l|l|l|l|l|}
\hline Plataforma / Biblioteca & $\begin{array}{l}\text { Autores } \\
\text { Impreso }\end{array}$ & $\begin{array}{c}\text { Titulos } \\
\text { Impreso }\end{array}$ & $\begin{array}{c}\text { Autores } \\
\text { Digital }\end{array}$ & $\begin{array}{c}\text { Títulos } \\
\text { Digital }\end{array}$ \\
\hline Amazon & 1728 & 7144 & 432 & 1101 \\
\hline Barnes \& Noble & 358 & 795 & 250 & 600 \\
\hline Biblioteca de São Paulo & 78 & 218 & 0 & 0 \\
\hline Biblioteca I. Lat. Berlín & 1479 & 4865 & 175 & 319 \\
\hline Biblioteca D. Unión Europea & 0 & 0 & 281 & 582 \\
\hline Biblioteca Nacional de España & 727 & 2473 & 522 & 1749 \\
\hline Biblioteca Nacional de Francia & 1186 & 3685 & 20 & 23 \\
\hline Biblioteca Nacional de Perú & 913 & 2855 & 9 & 10 \\
\hline Casa del Libro & 729 & 2211 & 198 & 512 \\
\hline Columbia University Libraries & 1257 & 4663 & 275 & 572 \\
\hline Free E-Books & 0 & 0 & 45 & 88 \\
\hline Gandhi & 231 & 666 & 240 & 617 \\
\hline Google Books & 2230 & 11140 & 92 & 143 \\
\hline iBook Store & 0 & 0 & 173 & 368 \\
\hline Kobo & 0 & 0 & 123 & 262 \\
\hline Librería Santa Fe & 548 & 1890 & 150 & 336 \\
\hline Livraria Cultura & 534 & 1785 & 337 & 1035 \\
\hline Nubico & 0 & 0 & 182 & 496 \\
\hline Perú Book Store & 36 & 76 & 45 & 154 \\
\hline Public Library New York & 1297 & 5760 & 8 & 21 \\
\hline Scribd & 0 & 0 & 715 & 1718 \\
\hline
\end{tabular}

FIGURA 2

Comparación de resultados de la oferta papel / digital por plataforma y biblioteca elaboración propia

En lo que hace a los resultados obtenidos en las búsquedas efectuadas por títulos literarios componentes del CLLyC (y los autores de estos), destacamos los resultados de los títulos que aparecen en más de 10 plataformas y bibliotecas (Figura 3. La columna"Fuentes" refiere a la cantidad de segmentos del CLLyC en que ubicamos el título; la columna "D" refiere a la cantidad de veces que fue hallado en digital; la columna"A" refiere a la cantidad de veces que fue hallado en soporte impreso). De los primeros 50 títulos que aparecen en soporte digital en las búsquedas realizadas, el $82 \%$ de ellos ( 41 títulos) pertenecen a autores cuyas obras están en dominio público. Esto ratifica que se transponen con mayor frecuencia aquellos títulos por los cuales no se pagan derechos. Así, solo dos títulos están presentes en 14 plataformas y bibliotecas: Crónica del Perú, de Pedro Cieza de León y Amalia, de José Mármol (obras que pertenecen al dominio público, en términos de derechos de autor). Luego, ocho títulos están presentes en 13 plataformas y bibliotecas. De ellos, seis títulos pertenecen al dominio público y solo uno es un título del canon de Bloom: El Aleph, de Jorge Luis Borges. El restante es la novela La tregua, del escritor uruguayo Mario Benedetti, cercana al bestseller dado que en América Latina se la relaciona con la película de Sergio Renán, que adoptó la novela como guion y se constituyó en el primer filme de la región en competir por un premio Oscar. El resto de los 50 títulos está presente en 10, 11 y 12 plataformas y bibliotecas. A su vez, de los primeros 50 títulos que más aparecen en digital cuatro son títulos bloomianos: tres son los volúmenes de cuentos de Jorge Luis Borges: El Aleph; Ficciones y El hacedor; además de Canto General de Pablo Neruda. Y, de esos 50 títulos, 20 son títulos de autores canónicos. A los ya mencionados se agregan títulos de los mismos Borges y Neruda y de los escritores bloomianos Gabriel García Márquez, Julio Cortázar, Miguel Ángel Asturias y Rubén Darío. De estos títulos, 
están en dominio público las seis obras pertenecientes a Rubén Darío. Asimismo, doce títulos con 10, 11 y 12 presencias en plataformas y bibliotecas son obras en dominio público de José Mármol, Rubén Darío, José Rodó, Cirilo Villaverde, López de Gómara, Alonso Ercilla, José Ingenieros, Inca Garcilaso, Francisco Cervantes de Salazar, Baldomero Lillo.

\begin{tabular}{|c|c|c|c|c|}
\hline Autor & Obra & Fuentes & D. & A. \\
\hline Cieza de León, Pedro & Crónica del Perú & 2 & 14 & 09 \\
\hline Mármol, José & Amalia & 2 & 14 & 10 \\
\hline Alberdi, Juan Bautista & Bases y puntos de partida para la... & 2,14 & 13 & 09 \\
\hline Alberdi, Juan Bautista & El crimen de la guerra & 2,14 & 13 & 11 \\
\hline Altamirano, Ignacio $\mathrm{M}$. & Clemencia & 2 & 13 & 12 \\
\hline Benedetti, Mario & La tregua & 2,14 & 13 & 12 \\
\hline Darío, Rubén & Prosas profanas & 2,3 & 13 & 09 \\
\hline Lillo, Baldomero & Sub Terra & 2,14 & 13 & 08 \\
\hline Villaverde, Cirilo & Cecilia Valdés & 2,14 & 13 & 12 \\
\hline Acevedo Díaz, Eduardo & Brenda & 14 & 12 & 04 \\
\hline Barros Arana, Diego & Historia general de Chile & 2,14 & 12 & 08 \\
\hline Blest Gana, Alberto & Martín Rivas & 14 & 12 & 10 \\
\hline Borges, Jorge Luis & El Aleph & $1,2,3,14$ & 12 & 13 \\
\hline Cruz, Sor Juana Inés de la & Obras & 2 & 12 & 11 \\
\hline Darío, Rubén & Cantos de vida y de esperanza & $2,3,14$ & 12 & 10 \\
\hline Ercilla y Zúñiga, Alonso de & La araucana & 2 & 12 & 09 \\
\hline Garcilaso de la Vega & Comentarios reales & 2 & 12 & 10 \\
\hline Ingenieros, José & El hombre mediocre & 2,14 & 12 & 10 \\
\hline Lillo, Baldomero & Sub sole & 2 & 12 & 08 \\
\hline López de Gómara, F. & Historia general de las Indias & 2 & 12 & 10 \\
\hline Rodó, José Enrique & Ariel & 2,14 & 12 & 10 \\
\hline Aguinis, Marcos & La gesta del marrano & 14 & 11 & 08 \\
\hline Asturias, Miguel Ángel & El señor Presidente & 2,14 & 11 & 12 \\
\hline Borges, Jorge Luis & Ficciones & $1,2,3,14$ & 11 & 12 \\
\hline Borges, Jorge Luis & Historia universal de la infamia & $2,3,14$ & 11 & 11 \\
\hline Cané, Miguel & Juvenilia & 2,14 & 11 & 12 \\
\hline Cieza de León, Pedro & Señorío de los Incas & 2 & 11 & 08 \\
\hline Darío, Rubén & Canto errante & 2 & 11 & 06 \\
\hline Darío, Rubén & Tierras solares & 2,14 & 11 & 07 \\
\hline Díaz del Castillo, Bernal & Historia verdadera de la conquista... & 2 & 11 & 11 \\
\hline García Márquez, Gabriel & La hojarasca & 2,14 & 11 & 08 \\
\hline García Márquez, Gabriel & La Mala Hora & 2,14 & 11 & 08 \\
\hline Güiraldes, Ricardo & Don Segundo Sombra & 2,14 & 11 & 08 \\
\hline Las Casas, Bartolomé de & Historia de las Indias & 2 & 11 & 06 \\
\hline Lugones, Leopoldo & Las fuerzas extrañas & 2,14 & 11 & 09 \\
\hline Montenegro, Carlos & Hombres sin mujer & 2,13 & 11 & 05 \\
\hline Neruda, Pablo & Canto general & $1,2,14$ & 11 & 12 \\
\hline Nervo, Amado & Los jardines interiores & 2,14 & 11 & 06 \\
\hline Nervo, Amado & Místicas & 2 & 11 & 07 \\
\hline Palma, Ricardo & Tradiciones & 2 & 11 & 12 \\
\hline Palma, Ricardo & Tradiciones peruanas & 2,14 & 11 & 12 \\
\hline Rabasa, Emilio & La bola & 2 & 11 & 09 \\
\hline Altamirano, Ignacio $\mathrm{M}$. & El Zarco & 2,14 & 10 & 12 \\
\hline Altamirano, Ignacio $\mathrm{M}$. & La Navidad en las montañas & 2,14 & 10 & 11 \\
\hline Arlt, Roberto & El juguete rabioso & 2 & 10 & 13 \\
\hline Borges, Jorge Luis & El Hacedor & $1,2,14$ & 10 & 10 \\
\hline Cané, Miguel & El viaje & 2 & 10 & 11 \\
\hline Carriego, Evaristo & Misas herejes & 2,14 & 10 & 10 \\
\hline Cervantes de Salazar, F. & Crónica de Nueva España & 2 & 10 & 08 \\
\hline Cortázar, Julio & Final del juego & 2 & 10 & 09 \\
\hline
\end{tabular}

FIGURA 3

Los 50 títulos del CLLyC que más aparecen en digital elaboración propia.

De esta manera, podemos interpretar de los resultados de las búsquedas que la oferta general en digital de literatura latinoamericana y caribeña se construye mediante la combinación de estrategias que provienen directamente de las estadísticas de ventas de los títulos en soporte impreso para asumir el riesgo empresario de su transposición a digital, lo que Cordón García (2004, p. 23) nomina como "demanda real de mercado". A ellas, se añaden estrategias relacionadas con los derechos de autor, estrategias de marketing y estrategias que provienen de la circulación de criterios estéticos y poéticas. Todas ellas sumadas a acciones del usuario mediante la incorporación de títulos ligados a su "gusto" (Figura 4). En este punto es necesario destacar que existen estrategias bibliotecológicas que permiten la incorporación de criterios de gusto del usuario. Si para Bourdieu (1988, p. 228), "el universo de los productos ofrecidos por cada uno de los campos de producción tiende a limitar de hecho el universo de las formas de la experiencia (estética, ética, política, etc.) que son objetivamente posibles en un momento dado", destacaremos que en el progreso de esa limitación, aquellas 
preferencias resultantes conforman un recorte de la oferta, que se manifiesta en el plano de la construcción del catálogo general de la literatura latinoamericana que se edifica por transposición al digital de los títulos que circulan en soporte impreso. Esos "posibles estilísticos" también inciden en la construcción tanto de la oferta de las plataformas como de la oferta de las bibliotecas, y esto en el marco de las estrategias adoptadas por los distintos agentes implicados en ambos sectores. Además, el modelo de adquisición de títulos impulsada por el usuario (Patron Driven Acquisition- $P D A$ ) es otra ventana que, subrayamos, también permitiría el ingreso de criterios de gusto de los lectores en la construcción de la colección. La estrategia de compras de títulos digitales basadas en el modelo de adquisición impulsada por el usuario (Cordón García et al., 2014; Davies, 2017; Esposito et al., 2012; Gammon y O’Neill, 2011; Vicente García, 2013; Walters, 2012) se constituye en un cambio paradigmático con la incorporación del usuario en la construcción de la colección e, igualmente, en una zona de negociación entre usuarios y bibliotecarios.

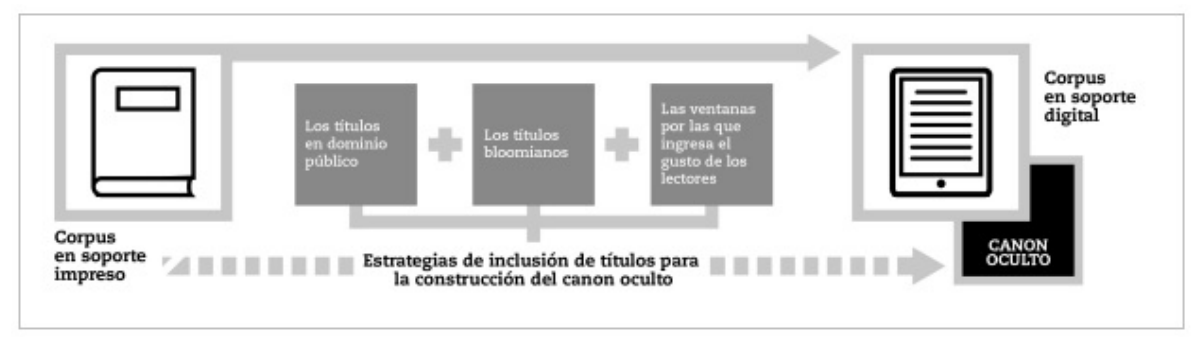

FIGURA 4

Estrategias de inclusión de títulos: la construcción del canon oculto en digital elaboración propia.

\section{D) Conclusiones}

La intervención de los usuarios en Scribd funciona como ventana para el ingreso de criterios poéticos, estéticos, de gusto (Bourdieu, 1988) en la construcción del catálogo general en digital del CLLyC, así como aquellas estrategias de compras de títulos digitales basadas en el modelo de adquisición impulsada por el usuario $(P D A)$, que dejan espacio a la participación directa del usuario en la construcción de la colección y se constituyen en una zona de negociación entre usuarios y bibliotecarios. De 6.447 títulos hallados en digital, Scribd ofrece 1.718 títulos, es decir, 35,48\% de los títulos y el 52,84\% de los autores existentes en digital para las búsquedas efectuadas. Luego de la Biblioteca Nacional de España (1.749 obras, 36,12\% de la oferta encontrada en digital), Scribd es la principal fuente de títulos hallados en las búsquedas. El listado de títulos que aparecen con mayor recurrencia en las tablas de resultados de las búsquedas (Figura 3) funciona de hecho como un canon oculto, resultante de la confluencia de estrategias editoriales y bibliotecológicas sumadas a acciones que incorporarían títulos mediante ventanas al gusto de los lectores y usuarios.

Asimismo, el corpus diseñado para las búsquedas se asienta en la aceptación de la porosidad e inestabilidad del sistema y en la inclusión de formas antagónicas y complementarias. De esta manera autoriza a ser replicado con fines investigativos diversos, dado que su configuración permite la supresión de algunas de las formas que lo componen y la inclusión de otras. Ahora bien, en el marco de la infinitud del corpus dada la vastedad de narrativas, poéticas y dispositividades de la literatura latinoamericana y caribeña, se detectan algunas perspectivas a incorporar como segmentos de este: los títulos autopublicados por sus autores; los títulos aportados por la literatura infantil y juvenil latinoamericana y caribeña; los títulos aportados por las literaturas religiosas de la región; los títulos aportados por manifestaciones culturales folklóricas de la región (tango, bossa, murga, reggae); los títulos y autores aportados por perspectivas tales como los estudios judeolatinoamericanos y los estudios afrolatinoamericanos; los títulos traducidos a otros idiomas; los títulos que se desarrollan en territorio de EE. UU. con la autodescripción de "literatura latinoamericana y caribeña"; los títulos en repositorios construidos por acción de los usuarios; las literaturas escritas en las lenguas de los 
pueblos originarios de la región; etc. Eagleton (1998, p. 22) afirma que "se deduce de la definición de literatura como forma de escribir altamente apreciada que no es una entidad estable". Esa inestabilidad se ha tomado en consideración para la construcción del CLLyC con la incorporación de formas sistémicas y extrasistémicas. Así, la inclusión de otras perspectivas (Figura 5) corroboraría la eficacia del diseño del CLLyC en el marco de la alteración de los segmentos que lo constituirían.

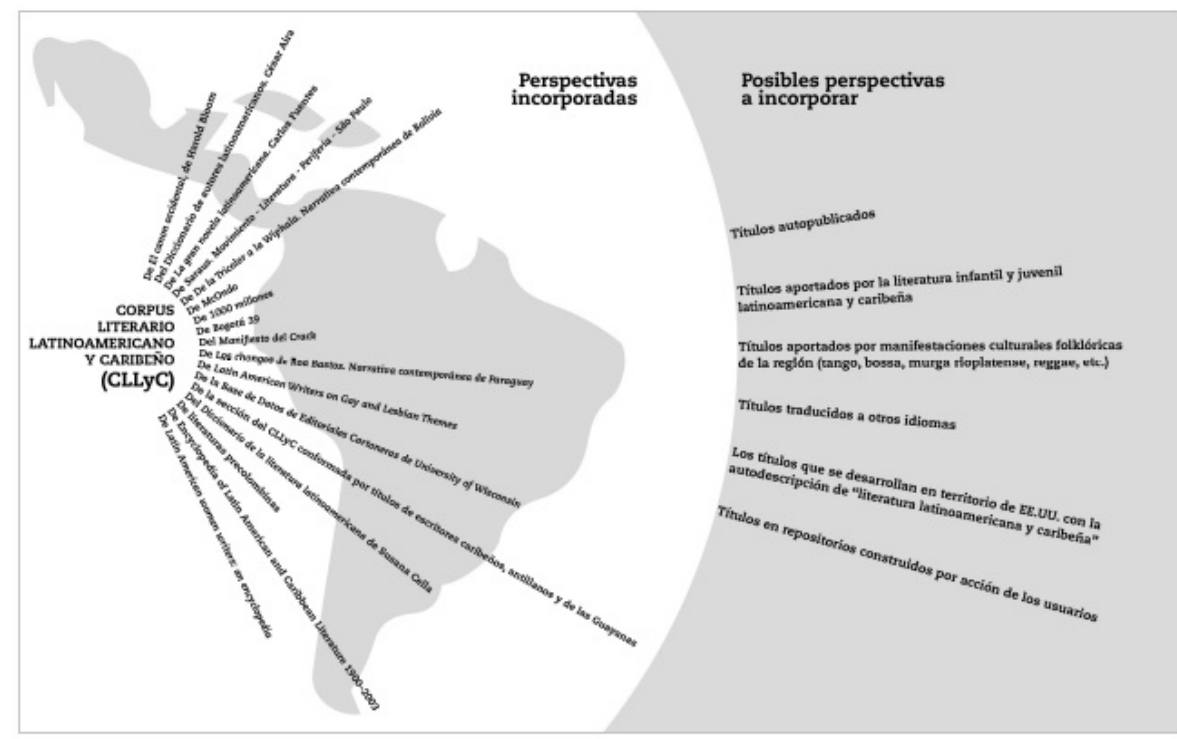

FIGURA 5

Perspectivas posibles a incorporar al CLLyC)

elaboración propia.

\section{REFERENCIAS}

Aira, C. (2001). Diccionario de autores latinoamericanos. Buenos Aires: Emecé y Ada Korn.

André, M. y Bueno, E. (2008). Latin American women writers: an encyclopedia. Nueva York: Routledge.

Ardao, A. (1996). Del hispanoamericanismo literario al latinoamericanismo literario. En La inteligencia latinoamericana (pp. 3-72). Montevideo: Universidad de la República.

Author Earnings (2017). February 2017 Big, Bad, Wide \& International Report: covering Amazon, Apple, B \& N, and Kobo ebook sales in the US, UK, Canada, Australia, and New Zealand. Recuperado de http://authorearnings.c om/report/february-2017/ [Consulta: 1/11/2017].

Balderston, D. y Gonzalez, M. (eds.) (2004). Encyclopedia of Latin American and Caribbean Literature 1900-2003. Londres-Nueva York: Routledge.

Benjamin, J., Kallicharan, L., McDonald, I. y Searwar, L. (eds.) (1998). They Came in Ships: An Anthology of IndoGuyanese Writing. Londres: Peepal Tree P.

Birbalsingh, F. (ed.) (1988).Jahaji Bhai: An Anthology of Indo-Caribbean Literature. Toronto: TSAR Publications. Bloom, H. (1995). El canon occidental. Barcelona: Anagrama.

Bogotá39 (s.f.). 39 escritores menores de 39. Hay Festival. Secretaría Distrital de Cultura, Recreación y Deporte de Bogotá. Recuperado de http://www.hayfestival.com/bogota39/es-index.aspx?skinid=7\&localesetting=es-ESac ysetting=EUR\&resetfilters=true [Consulta: $1 / 11 / 2017$ ].

Brown, S. y McWatt, M. (eds.) (2004). The Oxford Book of Caribbean Verse. Oxford, Inglaterra: Oxford UP.

Bourdieu, P. (1988). La distinción: criterios y bases sociales del gusto. Madrid: Taurus.

Cella, S. (1998). Diccionario de literatura latinoamericana. Buenos Aires: El Ateneo. 
Chartier, R. (2006). Inscribir y borrar. Cultura escrita y literatura (siglos XI-XVIII). Buenos Aires: Katz Editores.

Chowdhury, G. y Foo, S. (eds.) (2012). Digital libraries and information access: Research perspectives. Londres: Facet Publishing.

Cordón García, J. (2004). La visibilidad en los circuitos de la creación: literatura y traducción. En Gonzalo García, C. y García Yebra, V. (eds.), Manual de documentación y terminologia para la traducción especializada. Madrid: Arco/ Libros. Recuperado de http://eprints.rclis.org/8900/1/Cap_Visibilidad.pdf [Consulta: 30/10/2017].

Cordón García, J., Gómez Díaz, R., Alonso-Arévalo, J. y Alonso Berrocal, J. (2014). El ecosistema del libro electro\#nico universitario. Salamanca: Ediciones Universidad de Salamanca.

Davies, T. (2017). The evolution of an e-book demand-driven acquisition programme at Swinburne University of Technology. Insights, 30, 36-43. http://doi.org/10.1629/uksg.354 [Consulta: 30/10/2017].

Dedieu, H. (2013, febrero, 28). Measuring the iBook market. ASYMCO. Recuperado de http://www.asymco.com/2 013/02/28/measuring-the-ibook-market/ [Consulta: 30/10/2017].

Di Nucci, S., Recoaro, N. y Grieco y Bavio, A. (2014a). De la Tricolor a la Wiphala. Narrativa contemporánea de Bolivia. Buenos Aires: Santiago Arcos Editor.

Di Nucci, S., Recoaro, N. y Grieco y Bavio, A. (2014b). Los chongos de Roa Bastos. Narrativa contemporánea de Paraguay. Buenos Aires: Santiago Arcos Editor.

Donnell, A. y Bucknor, M. (eds.) (2011). The Companion to Anglophone Caribbean Literature. Londres: Routledge.

Donnell, A. y Lawson Welsh, S. (eds.) (1996). The Routledge Reader in Caribbean Literature. Londres: Routledge.

Eagleton, T. (1998). Introducción a la teoría literaria. México: Fondo de Cultura Económica.

Foster, David William (1991). Latin American Writers on Gay and Lesbian Themes: A Bio-Critical Sourcebook. Austin: University of Texas Press.

Fuentes, C. (2011). La gran novela latinoamericana. Madrid: Alfaguara.

Fuguet, A. y Gómez, S. (eds.) (1996). McOndo (una antología de nueva literatura hispanoamericana). Barcelona: Gijalbo-Mondadori.

Gammon, J. y O'Neill, E. (2011). OhioLINK-OCLC Collection \& Circulation Analysis Project 2011. Dublin, Ohio: OCLC Research. Recuperado de https://goo.gl/dGZy3H [Consulta: 1/3/2017].

Hall, S. (1991). The local and the Global: Globalization and Ethnicity. En King, A., Culture Globalization and the World-System. Contemporary Conditions for the Representation of Identity (pp. 19-39). Nueva York: Macmillan.

Helder, D., Henderson, D. y Orge, B. (2014). 1.000 millones: poesía en lengua española del siglo XXI. Rosario: Editorial Municipal de Rosario.

Klibanski, M. (2006). Grandes bibliotecas digitales y virtuales del mundo. Buenos Aires: portal Educ.ar. Recuperado de: http://portal.educ.ar/debates/educacionytic/super-sitios/grandes-bibliotecasdigitales-y-virtuales-del-mundo.php. [Consulta: 1/11/2017].

Krise, T. (ed.) (1999). Caribbeana: An Anthology of English Literature of the West Indies, 1657-1777. Chicago: University of Chicago Press.

Lotman, I. (1996). Para la construcción de una teoría de la interacción de las culturas (el aspecto semiótico). En La semiósfera. I. Semiótica de la cultura, del texto (pp. 61-76). Madrid: Cátedra.

Lotman, I. (2000). Sobre la dinámica de la cultura. En La semiósfera. III. Semiótica de las artes y de la cultura (pp. 194-214). Madrid: Cátedra.

Malamud, A. (2016). Entrevista personal, 29/01/15. En Vila, A. El canon oculto. La literatura de América Latina y el Caribe en la transposición al ecosistema digital (p.318). Salamanca: Ediciones Universidad de Salamanca.

Mignolo, W. (1998). Los cánones y (más allá de) las fronteras culturales (o ¿̇e quién es el canon del que hablamos?). En Sullá, E. (ed.), El canon literario (pp. 237-270). Madrid: Arco.

Palou, P. A., Urroz, E., Padilla, I., Chávez-Castañeda, R. y Volpi, J. (2000). Manifiesto Crack I, II, III, IV, V. Lateral. Revista de Cultura, 70. Recuperado de https://es.scribd.com/doc/64919561/Manifiesto-Crack [Consulta: 30/10/2017]. 
Pizarro, A. (coord.) (1987). Hacia una historia de la literatura latinoamericana. México: El Colegio de México/ Universidad Simón Bolívar.

Ramchand, K. (ed.) (1966). West Indian Narrative: An Introductory Anthology. Londres: Nelson.

Ramírez, M. (2016, agosto, 18). Kobo anuncia el Kobo Aura One. Todo Ereaders. Recuperado de https://www.todo ereaders.com/kobo-anuncia-el-kobo-aura-one.html [Consulta: 28/02/2018]

Rouquié, A. (1989). América Latina. Introducción al Extremo Occidente. México: Ed. Siglo Veintiuno.

Strickland, S. (2009). Born Digital: A Poet in the Forefront of the Field Explores What is - and is not - Electronic Literature. The Poetry Foundation. Recuperado de https://www.poetryfoundation.org/articles/69224/born-di gital [Consulta: 23/04/2017].

Tennina, L. (comp.) (2014). Antología Saraus. Movimiento - Literatura - Periferia - São Paulo. Buenos Aires: Tinta Limón.

Tramullas Saz, J. (2002). Propuestas de concepto y definición de la biblioteca digital. En e-LIS, 2002. Recuperado de http://eprints.rclis.org/15118/1/04_2002.pdf [Consulta: 23/10/2017].

Turiel, J. (2010). La manía de las listas: 100 bibliotecas digitales y 'recolectores' de libros electrónicos. Revista BID - textos universitaris de biblioteconomia i documentacio, 24. Recuperado de http://bid.ub.edu/24/turiel2.htm [Consulta: 13/05/2017].

University of Wisconsin (s.f.).Digital Collections. The Art Collection. Base de Datos de Editoriales Cartoneras. Madison: University of Wisconsin. Recuperado de http://digicoll.library.wisc.edu/WebZ/SearchOrBrowse?sessionid=0 1-53965-684142241 [Consulta: 4/1/2017].

Vicente García, R. (2013). Los libros electro\#nicos en las colecciones de las bibliotecas públicas. Revista bid textos universitaris de biblioteconomia i documentació, 30. Recuperado de http://bid.ub.edu/es/30/devicente.htm sthash. UPe1oIq8.dpuf [Consulta: 29/10/2017].

Walters, W. (2012). Patron-Driven Acquisition and the Educational Mission of the Academic Library. Piscataway: Rutgers University Libraries, Library Resources \& Technical Services, 56(3). Recuperado de http://alcts.ala.org/c cdablog/wp-content/uploads/2016/06/LC-2016-06.pdf [Consulta: 22/01/2017].

Walwyn, M. (ed.) (2001). Sugar Is All: Caribbean Short Stories and Poems from St. Kitts and Nevis. Kearney, Nebraska: Morris Publisher. 